\title{
Expansion of host range as a driving force in the evolution of Toxoplasma
}

\author{
John C Boothroyd \\ Department of Microbiology and Immunology, Stanford University School of Medicine, 94305-5124 Stanford CA, USA
}

\begin{abstract}
The apicomplexan parasite Toxoplasma gondii is unusual in being able to infect almost any cell from almost any warm-blooded animal it encounters. This extraordinary host-range contrasts with its far more particular cousins such as the various species of the malaria parasite Plasmodium where each species of parasite has a single genus or even species of host that it can infect. Genetic and genomic studies have revealed a key role for a number of gene families in how Toxoplasma invades a host cell, modulates gene expression of that cell and successfully evades the resulting immune response. In this review, I will explore the hypothesis that a combination of sexual recombination and expansion of host range may be the major driving forces in the evolution of some of these gene families and the specific genes they encompass. These ideas stem from results and thoughts published by several labs in the last few years but especially recent papers on the role of different forms of rhoptry proteins in the relative virulence of F1 Toxoplasma progeny in a particular host species (mice).
\end{abstract}

Key words: rhoptries - antigens - virulence - pathogenesis - recombination - genetics

Toxoplasma gondii is a protozoan parasite in the phylum Apicomplexa. As such, it is related to many wellknown pathogens such as those causing chicken coccidiosis (Eimeria) and malaria (Plasmodium). Like all of these organisms, Toxoplasma is exquisitely well adapted to a single definitive host, in this case felines, in which it undergoes its sexual cycle. Where Toxoplasma differs, however, is in its ability to infect an extremely wide range of intermediate hosts. In fact, it is found worldwide at a very high prevalence in many tens if not hundreds of warm-blooded animals ranging from birds through many mammals, even those who live exclusively in the sea. This makes Toxoplasma unique among eukaryotic infectious agents.

What is responsible for this extraordinary success? The answer to this is complex and includes many factors. First is the widespread presence of the definitive hosts (felines) across much of the earth from equatorial rain forests to tundra-like conditions in high elevations and extreme latitudes. This contrasts with the severe geographical restriction of suitable mosquito hosts for Plasmodium. Second, these cats can shed upwards of $10^{8}$ highly infectious and extremely stable oocysts into the environment making animals that graze or forage at risk of infection along with those who drink from contaminated water sources. Third, Toxoplasma has hit upon the magic molecular formula that enables it to productively infect almost any animal, once ingested, and become infectious to cats if the intermediate host falls prey to a feline. As discussed further below, this means it can recognize, attach, invade and reproduce within a host cell without regard to the species of that host. Not surpris-

Corresponding author: john.boothroyd@stanford.edu

Received 10 October 2008

Accepted 29 October 2008 ingly, this "generalist" ability also extends to cell type within a host species where again Toxoplasma shows remarkably little discrimination. At one point, infection of non-feline hosts might have been "accidental" and non-productive, i.e., evolution of a two-host life cycle likely came after a one-host (feline) cycle. The latter is the situation for most of the coccidian parasites related to Toxoplasma, especially the various species of Eimeria where there is only a single host species that serves as a natural host and with simple oral/fecal modes of transmission among members of that species.

This leads to the fourth, and maybe most remarkable trait of Toxoplasma: the infectivity of one intermediate host to another. This means that not only grazers who may accidentally ingest oocysts are potential hosts, but carnivores are as well. While many other scenarios can be imagined, it seems simplest to imagine the following path to reaching this current state: first Toxoplasma was a strict oral/fecal infectious agent of cats. Next, it gained the ability to infect non-felines but this was a dead-end (as it still is in many instances) because those animals were not in turn infectious to cats or other animals. The final and key piece was acquiring the ability to be transmitted beyond such hosts, either to other non-feline hosts or - and this would have the biggest numerical advantage - felines, themselves. This would convert an "accidental" host into a true "intermediate" host, as we see today.

A consequence of the above is that there is no theoretical reason why the parasite cannot persist in nature without an involvement of cats, moving up and down the food chain through carnivorism and scavenging. For example, oocysts from a cat could infect a foraging rat which is then eaten by a hawk which eventually dies and is eaten by a vulture which eventually dies and is eaten by a rat which is eaten by a hawk etc. Of course, the infected rat also stands a significant chance of being eaten by a cat and that event may produce millions of infectious oocysts with the potential to infect far more animals than a single (dead) intermediate host, but how these factors play out in 
nature is very hard to predict and probably highly dependent on many local variables - e.g., a single (dead) infected hyena might be food and thus a source of infection for many other potential intermediate hosts whereas a single infected cat might not shed its feces in an environment where the oocysts that are passed have the opportunity to be ingested or contaminate the water supply.

But, the above is all ground that has been well (and better) discussed by many others over the past four decades since the cat was first found to be the definitive host of Toxoplasma. And this article is not about ecolo$\mathrm{gy}$; it is about molecular biology and the question is how today is Toxoplasma able to productively infect such an extraordinary range of intermediate hosts and how might this have impacted the evolution of its genome? To consider all this, we need to first examine the detailed process of infection and the genes and proteins involved, at least as we now understand these things.

\section{The necessary pieces}

Ingestion - For productive infection of an intermediate host to occur, the following things must happen: first, the animal must ingest an infectious form (oocysts or tissue cysts) and this must survive the gastric juices and emerge able to infect the intestinal epithelium or nearby cells. Then the invaded parasite must replicate, infect more cells and ultimately differentiate into bradyzoites within tissue cysts. These must avoid clearance by the immune response and, finally, tissue containing such cysts must be eaten by another susceptible animal. Ideally, this next host will be a feline although, as stated above, infection of other so-called "intermediate" hosts represents a legitimate and viable ecological niche.

Regarding the first step, it is likely that the ability to survive the stomach is going to be relatively non-hostspecific, although ruminants, of course, may present a special problem (interestingly, although cattle are relatively refractory to infection, sheep, which are also ruminants, are fully susceptible making it unlikely that the rumen is the explanation for the refractoriness of cattle).

Attachment to a host cell - The first cells to be infected are not definitively known and could either be intestinal epithelial cells, cells within the lamina propria (assuming the parasite can actively transit the epithelium) or the rare cells that "sample" the intestinal lumen, e.g., in Peyer's patches. Until recently, highly sensitive methods have not existed to allow this question to be addressed with the necessary sensitivity, but previous methods have failed to detect infection of epithelial cells of mice infected with oocysts (Dubey et al. 1997).

It is not clear how contact with an appropriate host cell is sensed by the parasite but it could involve the GPIanchored surface antigens that are part of the SAG1-related sequence (SRS) family named for their homology to the first member of the family to be identified - surface antigen 1 (SAG1 or p30). The SRS genes encode an extensive family of related proteins with over 100 distinct $S R S$ genes in any given strain (Jung et al. 2004). This diversity is greatly expanded by the fact that for each of the more than $100 S R S$ loci, there is a very large number of alleles to be found in the many strains of Toxoplasma that exist in nature. Hence, within the species as a whole, there are probably many thousand distinct $S R S$ alleles genes that exist.

Although all appear to be structurally related, the SRS proteins differ in several important respects. First, some SRS are abundantly expressed while others are barely or not at all detectable (Lekutis et al. 2001). Second, the relative level of expression of the different $S R S$ loci varies between strains although this is a stable property of a given strain (i.e., there is no evidence for antigenic variation of the sort seen in Plasmodium). Third, some SRSs are exclusively found on a single developmental stage [e.g., SAG1 and SAG2A on tachyzoites (Dzierszinski et al. 2000, Lekutis et al. 2001)], SRS9 and SAG2C on bradyzoites (Cleary et al. 2002) or SporoSAG on sporozoites (Radke et al. 2004)), while a few appear to be common to at least two stages [(e.g., SAG3 on tachyzoites and bradyzoites (Dzierszinski et al. 2000)]. Fourth, some are highly immunogenic while others are not and this is not simply a result of their relative abundance. Fifth, although most SRS genes appear to be present in all strains, there are substantial sequence differences between strains with some loci missing or inactivated through having an in-frame sense codon in certain strains (Jung et al. 2004). Sixth, the SRS loci differ in the amount of polymorphism found; some are highly variable between strains while others are relatively constant, at least in the strains so far examined (http://toxodb.org/toxo).

It has been suggested that this diverse family serves at least two functions. First, they are adhesins/ligands that facilitate attachment of the parasite to the host cell surface (Mineo \& Kasper 1994). Their number, great diversity of sequence and variable levels of expression in different strains might then represent an adaptation to different hosts: for a given strain that has evolved for a given host species, there is a particular sequence and combination of SAGs that is optimal for attachment/invasion. A strain that experiences a chance gene duplication followed by some mutation to a different sequence might find itself better suited than its ancestors to a new host species that it might encounter. This would represent a powerful selection for expansion and sequence drift in the SRS family.

The second suggested role of SAGs has been as immune-modulators (Rachinel et al. 2004, Kim \& Boothroyd 2005, Kim et al. 2007). This notion supposes that certain SRSs (e.g., SAG1 and SAG2A) that are tachyzoitespecific draw the immune response specifically toward them and away from other crucial SAGs whose function requires them not to be interfered with (by, for example, excessive antibody binding which might block invasion). According to the hypothesis being explored here, the high degree of sequence variability and strain-specific differences in repertoire and relative abundance of the SRSs might allow strains that have an altered repertoire of SRS expression to more effectively manipulate or evade the immune response in a newly encountered host species.

Recently, another family of GPI-anchored proteins has been described (Pollard et al. 2008). These have been dubbed the SUSA family (for SAG1-unrelated surface 
antigens) and they number about 31 known genes. The function of these proteins is completely unknown but they too appear to be developmentally regulated (one is particularly abundant on bradyzoites) and thus all that was described above for SRS genes could equally apply to this gene family (although there are as yet no data to indicate that these proteins have a role in attachment/invasion).

Getting in - Following attachment, parasites actively enter into the host cell using a group of proteins that originate from the rhoptry necks and are referred to as the RON proteins (Alexander et al. 2005, Lebrun et al. 2005). These collaborate with a micronemal protein, AMA1, to form the ring-like moving junction where the invading parasite forms a ring of contact with the host cell's plasma membrane. Interestingly, these genes are not highly variable between strains and are not each part of a large gene family. This is consistent with the moving junction performing a role that is not highly specialized to the host being infected: once specific contact is made, one cell is much the same as another in terms of the machinery necessary to drive the parasite in [e.g., Ford may sell different exterior shapes and colors of passenger cars in different countries, to suit local (host) tastes but the underlying chassis, engine, transmission etc. is often exactly the same]. Hence, host range may not have been a major force in the evolution of the RON and AMA1 proteins and, hence, the reasons why they are not present in high copy number in the Toxoplasma genome and not strikingly variable in sequence. That said, there are two copies of AMA1 and several of the RON genes suggesting that there has been some selection for a modest duplication. Since essentially all of the studies on RONS and AMA1 have involved tachyzoites, it could be that the other paralogue for each of these genes is involved in invasion by one of the other life cycle stages, such as bradyzoites or the sexual stages. But host range seems unlikely to be at play.

Taking over - The Toxoplasma rhoptries have long been known to release their contents during invasion, apparently filling the parasitophorous vacuole (PV) space and/or coating the PV membrane. Provocative images by Kimata and Tanabe (1987) provided the first indication that rhoptry proteins (ROPs) might actually enter the host cell in vesicular or free form. Hakansson et al. (2001) next showed that ROPs are present as beads on a string or "evacuoles" that extend outwards from a parasitophorous vacuole, sometimes connecting two vacuoles with a long, filament-like structure. These appear to be the means by which proteins can flow from one PV to another. More recent reports have shown that at least two ROPs, a putative protein kinase [ROP16 (Saeij et al. 2007)] and a protein phosphatase [PP2C-hn (Gilbert et al. 2007)] are introduced into the host cell - during or very shortly after invasion - as freely soluble, cytosolic proteins that can migrate to the nucleus (using nuclear import signals recognized by the host import machinery).

The ROPs described above originate from the bulbous rhoptry base and a majority of these are part of a large family of proteins with homology to protein kinases and typified by the archetypal member ROP2 (El Hajj et al. 2006). The ROP2 family is extensive with at least 25 copies (paralogues) being carried by a given strain (http://toxodb.org/toxo). Often these are repeated with similar but non-identical paralogues being found in a tandem array. There is tremendous allelic diversity at many of these loci with, for example, ROP18 being one of the most divergent loci when comparing type I, II and III strains (Saeij et al. 2006, Taylor et al. 2006).

Some members of the ROP2 family encode proven functional kinases while a majority seems to encode devolved proteins where the kinase function has been lost (El Hajj et al. 2006). Among the apparently active kinases, ROP16 and ROP18 have been shown to play a key role in interaction with the host cell (El Hajj et al. 2007) and/or host animal (Saeij et al. 2006, Taylor et al. 2006). ROP16 somehow intersects STAT3 and STAT6, host transcription factors that are central to the immune response (Saeij et al. 2007). The ROP18 targets are unknown but, as with ROP16, there are major allelic differences between these genes in different strains of Toxoplasma with consequent differences in the nature and degree of infection. The ROP18 differences include both the sequence and relative abundance of the transcript (and protein produced); type I strains produce several orders of magnitude more ROP18 transcript than type III strains. These differences in ROP18 manifest in enormous differences in $\mathrm{LD}_{50}$ and introducing a different ROP18 allele into a given strain can changes its virulence in mice by over 10,000-fold (Saeij et al. 2006, Taylor et al. 2006).

The host range hypothesis posits that the expansion of the ROP2 family and the extreme divergence in sequence of its component members is the result of the importance of this family in the ability to productively infect a new host species. The benefit in this case would come from expressing a member of this family that interacts with the host machinery in just the right way resulting in maximal transmission. In the case of ROP16, for example, a given allelic form of the protein might result in so much STAT3 activation that IL12 levels are depressed to the degree that the host is overwhelmed by the infection and dies before infectious tissue cysts form. In another host, that same allelic form of ROP16 might cause too little activation of STAT3 and thus excessive IL12 that again resulted in premature host death, but this time through a cytokine storm. Only in a particular set of host species is the activity of a particular allele of ROP16 "just right", producing a chronically infected host that can go on to infect other hosts. The same might be true for ROP18 although the mechanism by which different allelic forms impact virulence has yet to be determined.

The extent of allelic variation at a given locus as a function of time will be limited such that it may be very unlikely that the gene can acquire the mutations necessary for infection of a novel host species in a new ecological niche that the parasite "suddenly" (in evolutionary terms) encounters. This could be because the rate of acquiring new mutations is too slow and/or such mutations result in the loss of infectivity to a species of host that is otherwise still a "good" host for the parasite (i.e., mutating to be able to infect a house sparrow may not be evolutionarily advantageous if it comes at the cost of 
ability to infect a house mouse). A solution to these problems could come through expansion of the gene family: it increases the chances of happening on the "just right" activity because there is more sequence space being explored when more genes are evolving in parallel and it does not necessarily impact infectivity for an existing host species.

Of course these genes are unlikely to operate independently of each other. The ultimate expression of even a single protein in a vertebrate cell is a complex interplay of many pathways that collectively produce the desired levels. Modulating such complexity may require a large number of parasite factors that cooperate to achieve a new level of host gene expression. For example, and this may be especially true for the ROP2 family with its many "non-catalytic" members, several ROP2-like genes may act in concert producing a particular blend that achieves the "just right" activity when expressed together. The advantage would be similar to that for a group of artists who have to match the color of a sunset who are told to mix paints from different tubes compared to those who must use only one tube of a paint at a time; the chance of one individual hitting on the right hue is massively higher in the group that is blending the colors.

But how to achieve the right blend? Doing so by stepwise mutation and/or expansion of the gene family would clearly be far too slow to allow the kind of rapid adaptation to new circumstances that appears to have occurred in recent times for Toxoplasma. There is a simple solution to this problem: sexual recombination. Because a single cat can release upwards of $10^{8}$ recombinant F1 progeny (assuming the cat was not previously infected with Toxoplasma and that it was simultaneously infected with similar levels of two strains), each expressing a possibly unique mix of ROP2 family genes, there is a very good chance that one or more will have close to or exactly an optimal mix for a new host.

Indeed, recent studies with experimental crosses between different strain types confirm that the interaction of the F1 progeny with a given host (mice) can vary enormously; e.g., their virulence can span over five orders of magnitude when simply measuring $\mathrm{LD}_{50}$ (Grigg et al. 2001, Saeij et al. 2006, 2007, Taylor et al. 2006) . In a host species that the parasite is encountering for the first time, such a range will greatly improve the chances of there being at least one strain that is well suited to productive infection of (and subsequent transmission from) a new host. These differences appear to be due, in large part, to allelic differences in the ROP2 family of genes.

Rhoptries are not the only organelle with a role in taking over the host cell. Dense granules also secrete their contents during and after invasion. These so-called "GRA" proteins represent an interesting contrast between the divergent/expanded $R O P 2$ genes and the highly monomorphic RONs. GRA6, for example, is present as a unique gene but appears to be under considerable evolutionary pressure, perhaps because it is a major Tcell antigen (Blanchard et al. 2008). Drift of the sequence may enable the resulting parasite to better avoid immune detection because the resulting peptide cannot be efficiently presented by the particular MHC of the infected host. In mice, for example, the C-terminus of GRA6 is a major T-cell epitope but only if the mouse has the $\mathrm{L}_{\mathrm{d}}$ allele for class I MHC; other combinations of GRA6 and MHC-I alleles may not yield efficient presentation. Of course, whether this is to the advantage of Toxoplasma will depend on the rest of the many other interactions it has with its host and, specifically, whether more or less immune recognition would bring it closer to its optimum with that host.

Increasing the odds - In addition to expanding the host range, sequence drift of important antigens could provide a substantial advantage to the species as a whole in allowing one strain to infect a host that is already infected by another, antigenically distinct strain. This provides the simple advantage of giving different Toxoplasma strains access to more individuals within a given host species. Perhaps more importantly, this might be key for enabling at least moderately efficient sexual recombination; for sexual recombination to occur, a feline must become infected at essentially the same time with two different strains of Toxoplasma. Sequential infection won't yield efficient mating because gametogenesis peaks within a fairly tight window (1-2 days) after the cat becomes infected and so if strain B infects the cat two days after strain A, their gametes are generally not present at the same time and so mating will likely be rare if at all. Felines are true carnivores and they ingest prey only when their hunt is successful; the chances of ingesting two prey in rapid succession that are infected with different strains are probably low. A far more efficient solution for the parasite would be if a given intermediate host was concomitantly infected with two strains so that timing is not an issue and efficient interstrain-mating is far more likely. This may be the most important evolutionary advantage to super-infection.

\section{Clues from population biology}

Studies over the last two decades have shown that in many regions, a select number of strains dominate in certain host species, especially humans and their domesticated animals/livestock (Darde et al. 1988, Sibley \& Boothroyd 1992, Howe \& Sibley 1995, Ajzenberg et al. 2002, 2004, Miller et al. 2004, Lehmann et al. 2006). This appears to have been the result of a clonal expansion of a small number of extremely fit genotypes. Most extraordinarily, in Europe and North America, the three dominant types appear to all be closely related and a result of just one or two crosses (meaning, just one or two cats!) between two or, perhaps, three founding lines (Boyle et al. 2006).

What properties do these superfit strains possess that others lack? A major clue is that experimental crosses yield progeny with enormous variation in their interaction with the host (Grigg et al. 2001) and these differences are largely due to different allelic combinations at $R O P$ genes. Given this, it seems likely that in nature, selection has yielded a few strains that have the right mix of alleles at key ROP and, perhaps, GRA, SRS, SUSA and other loci that have allowed these strains to dominate new ecological niches. In the case of Toxoplasma, such 
niches are likely to be newly encountered, abundant host species - for example, the large numbers of rodents that are now living in close association with people and, more importantly, their feline companions. Tantalizingly, one of the smallest chromosomes ("1A") appears to carry some of the most important genes although exactly which loci has yet to be determined (Khan et al. 2006).

The model presented here incorporates data from more global analyses that show the diversity of strains when regions outside of Europe and North America, and/or when hosts beyond livestock and humans, are examined (Ajzenberg et al. 2002, 2004, Khan et al. 2006, 2007, Lehmann et al. 2006, Sibley \& Ajioka 2008). These strains are presumably more "natural" and are existing within the true complexity that exists in less disturbed ecosystems. Interestingly, so far South America seems to have the greatest diversity of strains and this may reflect the ancient geographic origins of Toxoplas$m a$. Alternatively, the greater diversity seen for virtually all biological niches in the tropics may be operating with Toxoplasma, as well; that is, the relative richness of potential hosts (and routes of transmission?) that exists within the tropics may have resulted in a correspondingly more diverse range of genotypes of the parasite that can co-exist in such an environment. Clearly, a careful examination of African isolates will help discriminate between these alternative explanations and such analyses are anxiously awaited.

\section{What about the definitive host?}

The above has focused on the selection that operates on different genotypes at the level of intermediate host range. What about the definitive hosts? Might they not also be exerting some selective pressure? Clearly, differences exist between a domestic house cat and a lion that might make a given Toxoplasma genotype better suited to one of those species than the other. Might this situation even extend outside felines? In other words, might some strains of Toxoplasma be adapted to sexual replication in non-feline hosts? How many strains have actually been tested for their ability to infect non-felines? There is a good body of data to implicate cats as the major host in well-studied regions and with well-studied strains of the parasite but what about strains from regions and ecological niches only recently investigated. If only to know that we are not missing something big, and in light of all that we now know and with the help of all of the tools that are now available, perhaps it is time to revisit the "felines-only" dogma.

This review has attempted to synthesize the results and thinking of many individuals and has little in it that is truly new. An enormously broad host range is, of course, a long-recognized aspect of Toxoplasma biology. So, too, is the clonal population structure of the parasite. The emphasis I am trying to make here is that once Toxoplasma developed the ability to infect a few hosts, and given its multiple potential modes of transmission, there was nothing to prevent it from spreading to a colossally large number of hosts, so long as it had the right mix of proteins to "engage" that host without overwhelming it. A little gene duplication, a little time for some mutations and, most of all, a little sexual recombination to offer up all the possible combinations and, voila, you have the perfect strain for a new niche. Toxoplasma appears to have just such a phenomenally successful formula that may explain it being one of the most successful infectious agents known.

\section{ACKNOWLEDGEMENTS}

To my many colleagues in and out of the lab, for helpful discussions related to the subject of this review, in particular Jim Ajioka, Daniel Ajzenberg, Dave Alexander, Jon Boyle, Marie-Laure Darde, Jean-Francois Dubremetz, JP Dubey, David Ferguson, Michael Grigg, Tovi Lehmann, Steve Parmley, Jeroen Saeij and David Sibley, all contributed to or even first articulated the notions presented here.

\section{REFERENCES}

Ajzenberg D, Banuls AL, Su C, Dumetre A, Demar M, Carme B, Darde ML 2004. Genetic diversity, clonality and sexuality in Toxoplasma gondii. Int J Parasitol 34: 1185-1196.

Ajzenberg D, Banuls AL, Tibayrenc M, Darde ML 2002. Microsatellite analysis of Toxoplasma gondii shows considerable polymorphism structured into two main clonal groups. Int J Parasitol 32: 27-38.

Alexander DL, Mital J, Ward GE, Bradley P, Boothroyd JC 2005. Identification of the moving junction complex of Toxoplasma gondii: a collaboration between distinct secretory organelles. PLoS Pathog 1: e17.

Blanchard N, Gonzalez F, Schaeffer M, Joncker NT, Cheng T, Shastri AJ, Robey EA, Shastri N 2008. Immunodominant, protective response to the parasite Toxoplasma gondii requires antigen processing in the endoplasmic reticulum. Nat Immunol 9: 937-944.

Boyle JP, Rajasekar B, Saeij JP, Ajioka JW, Berriman M, Paulsen I, Roos DS, Sibley LD, White MW, Boothroyd JC 2006. Just one cross appears capable of dramatically altering the population biology of a eukaryotic pathogen like Toxoplasma gondii. Proc Natl Acad Sci USA 103: 10514-10519.

Cleary MD, Singh U, Blader IJ, Brewer JL, Boothroyd JC 2002. Toxoplasma gondii asexual development: identification of developmentally regulated genes and distinct patterns of gene expression. Eukaryot Cell 1: 329-340.

Darde ML, Bouteille B, Pestre-Alexandre M 1988. Isoenzymic characterization of seven strains of Toxoplasma gondii by isoelectrofocusing in polyacrylamide gels. Am J Trop Med Hyg 39: 551-558.

Dubey JP, Speer CA, Shen SK, Kwok OC, Blixt JA 1997. Oocyst-induced murine toxoplasmosis: life cycle, pathogenicity and stage conversion in mice fed Toxoplasma gondii oocysts. J Parasitol 83: 870-882.

Dzierszinski F, Mortuaire M, Cesbron-Delauw MF, Tomavo S 2000. Targeted disruption of the glycosylphosphatidylinositol-anchored surface antigen SAG3 gene in Toxoplasma gondii decreases host cell adhesion and drastically reduces virulence in mice. $\mathrm{Mol} \mathrm{Mi-}$ crobiol 37: 574-582.

El Hajj H, Demey E, Poncet J, Lebrun M, Wu B, Galeotti N, Fourmaux MN, Mercereau-Puijalon O, Vial H, Labesse G, Dubremetz JF 2006. The ROP2 family of Toxoplasma gondii rhoptry proteins: proteomic and genomic characterization and molecular modeling. Proteomics 6: 5773-5784.

El Hajj H, Lebrun M, Arold ST, Vial H, Labesse G, Dubremetz JF 2007. ROP18 is a rhoptry kinase controlling the intracellular proliferation of Toxoplasma gondii. PLoS Pathog 3: e14. 
Gilbert LA, Ravindran S, Turetzky JM, Boothroyd JC, Bradley PJ 2007. Toxoplasma gondii targets a protein phosphatase $2 \mathrm{C}$ to the nuclei of infected host cells. Eukaryot Cell 6: 73-83.

Grigg ME, Bonnefoy S, Hehl AB, Suzuki Y, Boothroyd JC 2001. Success and virulence in Toxoplasma as the result of sexual recombination between two distinct ancestries. Science 294: 161-165.

Hakansson S, Charron AJ, Sibley, LD 2001. Toxoplasma evacuoles: a two-step process of secretion and fusion forms the parasitophorous vacuole. Embo J 20: 3132-3144.

Howe DK, Sibley LD 1995. Toxoplasma gondii comprises three clonal lineages: correlation of parasite genotype with human disease. $J$ Infect Dis 172: 1561-1566.

Jung C, Lee CY, Grigg ME 2004. The SRS superfamily of Toxoplasma surface proteins. Int J Parasitol 34: 285-296.

Khan A, Bohme U, Kelly KA, Adlem E, Brooks K, Simmonds M, Mungall K, Quail MA, Arrowsmith C, Chillingworth T, Churcher C, Harris D, Collins M, Fosker N, Fraser A, Hance Z, Jagels K, Moule S, Murphy L, O'Neil S, Rajandream MA, Saunders D, Seeger K, Whitehead S, Mayr T, Xuan X, Watanabe J, Suzuki Y, Wakaguri H, Sugano S, Sugimoto C, Paulsen I, Mackey AJ, Roos DS, Hall N, Berriman M, Barrell B, Sibley LD, Ajioka JW 2006. Common inheritance of chromosome Ia associated with clonal expansion of Toxoplasma gondii. Genome Res 16: 1119-1125.

Khan A, Fux B, Su C, Dubey JP, Darde ML, Ajioka JW, Rosenthal BM, Sibley LD 2007. Recent transcontinental sweep of Toxoplasma gondii driven by a single monomorphic chromosome. Proc Natl Acad Sci USA 104: 14872-14877.

Kim SK, Boothroyd JC 2005. Stage-specific expression of surface antigens by Toxoplasma gondii as a mechanism to facilitate parasite persistence. J Immunol 174: 8038-8048.

Kim SK, Karasov A, Boothroyd JC 2007. Bradyzoite-specific surface antigen SRS9 plays a role in maintaining Toxoplasma gondii persistence in the brain and in host control of parasite replication in the intestine. Infect Immun 75: 1626-1634.

Kimata I, Tanabe K 1987. Secretion by Toxoplasma gondii of an antigen that appears to become associated with the parasitophorous vacuole membrane upon invasion of the host cell. J Cell Sci 88: 231-239.

Lebrun M, Michelin A, El Hajj H, Poncet J, Bradley PJ, Vial H, Dubremetz JF 2005. The rhoptry neck protein RON4 re-localizes at the moving junction during Toxoplasma gondii invasion. Cell Microbiol 7: 1823-1833.
Lehmann T, Marcet PL, Graham DH, Dahl ER, Dubey JP 2006. Globalization and the population structure of Toxoplasma gondii. Proc Natl Acad Sci USA 103: 11423-11428.

Lekutis C, Ferguson DJ, Grigg ME, Camps M, Boothroyd JC 2001. Surface antigens of Toxoplasma gondii: variations on a theme. Int J Parasitol 31: 1285-1292.

Miller MA, Grigg ME, Kreuder C, James ER, Melli AC, Crosbie PR, Jessup DA, Boothroyd JC, Brownstein D, Conrad PA 2004. An unusual genotype of Toxoplasma gondii is common in California sea otters (Enhydra lutris nereis) and is a cause of mortality. Int J Parasitol 34: 275-284.

Mineo JR, Kasper LH 1994. Attachment of Toxoplasma gondii to host cells involves major surface protein, SAG-1 (P30). Exp Parasitol 79: 11-20.

Pollard AM, Onatolu KN, Hiller L, Haldar K, Knoll LJ 2008. Highly polymorphic family of glycosylphosphatidylinositol-anchored surface antigens with evidence of developmental regulation in Toxoplasma gondii. Infect Immun 76: 103-110.

Rachinel N, Buzoni-Gatel D, Dutta C, Mennechet FJ, Luangsay S, Minns LA, Grigg ME, Tomavo S, Boothroyd JC, Kasper LH 2004. The induction of acute ileitis by a single microbial antigen of Toxoplasma gondii. J Immunol 173: 2725-2735.

Radke JR, Gubbels MJ, Jerome ME, Radke JB, Striepen B, White MW 2004. Identification of a sporozoite-specific member of the Toxoplasma SAG superfamily via genetic complementation. Mol Microbiol 52: 93-105.

Saeij JP, Boyle JP, Coller S, Taylor S, Sibley LD, Brooke-Powell ET, Ajioka JW, Boothroyd JC 2006. Polymorphic secreted kinases are key virulence factors in toxoplasmosis. Science 314: 1780-1783.

Saeij JP, Coller S, Boyle JP, Jerome ME, White MW, Boothroyd JC 2007. Toxoplasma co-opts host gene expression by injection of a polymorphic kinase homologue. Nature 445: 324-327.

Sibley LD, Ajioka JW 2008. Population structure of Toxoplasma gondii: clonal expansion driven by infrequent recombination and selective sweeps. Annu Rev Microbiol 62: 329-351.

Sibley LD, Boothroyd JC 1992. Virulent strains of Toxoplasma gondii comprise a single clonal lineage. Nature 359: 82-85.

Taylor S, Barragan A, Su C, Fux B, Fentress SJ, Tang K, Beatty WL, Hajj HE, Jerome M, Behnke MS, White M, Wootton JC, Sibley LD 2006. A secreted serine-threonine kinase determines virulence in the eukaryotic pathogen Toxoplasma gondii. Science 314: $1776-1780$. 